\title{
Retrospective Evaluation of Thromboembolism Risk in Ovarian Cancer Patients Treated with Bevacizumab
}

\author{
Anna Kuk ${ }^{1} \cdot$ Magdalena Magnowska $^{1} \cdot$ Wiktor Suchy $^{1} \cdot$ Joanna Swierczynska $^{1}$. \\ Mikolaj Piotr Zaborowski $^{1}$ - Michal Gaca ${ }^{2}$ - Ewa Nowak-Markwitz ${ }^{1}$
}

Published online: 4 June 2017

(C) The Author(s) 2017. This article is an open access publication

\begin{abstract}
Background Bevacizumab is used in addition to standard, platinum-based chemotherapy to treat advanced-stage ovarian cancer patients. Thrombosis is a well-documented adverse effect of bevacizumab.

Objective The aim of this study was to identify predictive parameters for thromboembolic events in ovarian cancer patients and to explain how bevacizumab increases the risk of these events.

Patients and Methods Fifty-seven FIGO stage III ovarian cancer patients who underwent cytoreductive surgery and chemotherapy were identified and included in this retrospective study. Twenty-six patients were treated with carboplatin and paclitaxel (CP) only (control group), and 31 patients received $\mathrm{CP}$ with bevacizumab (study group). The two groups were compared with regard to thrombosis risk factors and laboratory parameters (total leukocytes, platelet count, hemoglobin, APTT, prothrombin time, INR, fibrinogen levels, D-dimer concentration) before treatment, after each course of chemotherapy, and during thromboembolic events.

Results Only patients in the group receiving bevacizumab experienced venous thromboembolism (VTE) ( $p=0.03, \chi^{2}$ test). VTE occurred on average at the 13th cycle of chemotherapy. Patients who experienced VTE had increased BMI before chemotherapy as compared to patients with no thromboembolic
\end{abstract}

Anna Kuk

aniak92@gmail.com

1 Department of Gynecology, Obstetrics and Gynecologic Oncology, Division of Gynecologic Oncology, Poznan University of Medical Sciences, ul.Polna 33, 60-535 Poznan, Poland

2 Department of Anesthesiology in Obstetrics and Gynecology, Poznan University of Medical Sciences, ul.Polna 33, 60-535 Poznan, Poland event (27.2 vs. $23.3, \mathrm{p}=0.005$, Mann-Whitney test). D-dimer concentration before treatment was also elevated more in patients affected by VTE (3132.5) than in the non-VTE group (956.43) ( $p=0.0007$, Mann-Whitney test). During the first four administrations of chemotherapy in patients with future VTE, there was a reduction in D-dimer concentration and an extension of APTT. A D-Dimer level higher than $485 \mathrm{ng} / \mathrm{mL}$ prior to first chemotherapy indicates for a risk of VTE with $94 \%$ sensitivity and $36 \%$ specificity.

Conclusions An elevated D-dimer level and high BMI before chemotherapy are risk factors for VTE in ovarian cancer patients receiving bevacizumab. Bevacizumab possibly increases the risk for VTE.

\section{Key Points}

An elevated D-dimer level and high BMI before chemotherapy are risk factors of venous thromboembolism (VTE) in ovarian cancer patients.

Only patients in the group receiving bevacizumab experienced VTE.

\section{Introduction}

Venous thromboembolism (VTE) is a major complication in oncology, caused both by the cancer itself and by chemotherapy $[1,2]$. Cancer disease increases the VTE risk $>$ fourfold, and with chemotherapy this further increases to >sixfold compared to healthy non-cancer patients [3]. One out of five patients with cancer will develop a thromboembolic event [4]. Ovarian cancer increases the risk of thromboembolic events only 
moderately [odds ratio (OR): $3.1 ; 95 \%$ confidence interval (CI): 0.6-15.3] compared to lung cancer, hematological cancers, and gastrointestinal cancers (OR of 20 and more) [5].

Thromboembolic events are difficult to predict and prevent, as there are currently no laboratory indicators with predictive values with satisfactory effectiveness. There are also no clear recommendations as to which patients are most susceptible to thromboembolic events. ASCO guidelines do not recommend routine thromboprophylaxis in the outpatient setting; however, the guidelines do suggest assessing the risk for VTE. The Khorana risk score for the prediction of VTE is proposed as a method to assess this risk. The score includes a combination of easily available clinical and laboratory variables, such as BMI $\geq 35 \mathrm{~kg} / \mathrm{m}^{2}$; total leukocytes $>11 \times 10^{9} / \mathrm{L}$; platelet count $\geq 350 \times 10^{9} / \mathrm{L}$; Hemoglobin concentration $<100 \mathrm{~g} / \mathrm{L}$, or erythropoietin use. At the cutoff point for high risk (score $\geq 3$ ) it has a negative predictive value (probability of no VTE in those designated low risk) of $98.5 \%$, a positive predictive value (probability of VTE in those designated high risk) of $7.1 \%$, a sensitivity (probability of high risk in those experiencing VTE) of $40.0 \%$, and a specificity (probability of low risk in those not experiencing VTE) of $88 \%$ in the derivation cohort [6]. This suggests that the score is effective for determining only those at low risk and that additional variables are likely needed to identify the high risk patients.

Ovarian cancer is usually diagnosed in advanced stages (more than half of all cases as late as FIGO stage III), when the tumor has invaded adjacent structures in the pelvis and abdomen, and metastases have spread to other parts of the body. This is known to considerably increase the risk of thromboembolic events. Currently, bevacizumab, a monoclonal antibody against vascular endothelial growth factor (VEGF) is registered in the EU and USA and used in addition to standard, platinum-based chemotherapy to treat advanced-stage (FIGO IIIb and higher) ovarian cancer patients. Bevacizumab has been proven to prolong the median-progression free survival in patients with advanced (FIGO stage III and IV) ovarian cancer [7] and to increase tumor response in platinum-resistant epithelial ovarian cancer in phase II $[8,9]$ and phase III trials [10]. One of the drug's well-documented side effects is thrombosis [11]. Various studies [11, 12] suggest that combining bevacizumab with standard chemotherapy significantly increases the risk of thromboembolic disease, while other research seem to negate this claim [13, 14]. Moreover, most studies and meta-analyses actually show that bevacizumab significantly increases arterial thromboembolism only, not VTE $[12,15]$.

The aim of our present study was to check which of the parameters assessed in our study (total leukocytes, platelet count, hemoglobin, APTT, prothrombin time, INR, fibrinogen levels, D-dimer concentration) are most valuable in predicting thromboembolic events and to explain the way bevacizumab increases the risk for these events.

\section{Patients and Methods}

\subsection{Patients}

Fifty-seven FIGO stage III ovarian cancer patients who underwent cytoreductive surgery and chemotherapy were identified and included in this retrospective study. The control group (26 patients) was treated with three to six cycles of standard first line chemotherapy consisting of carboplatin (AUC 6 i.v.) and paclitaxel (175 mg/m ${ }^{2}$ i.v.) (CP). The study group (31 patients) was treated with $\mathrm{CP}$ chemotherapy with the addition of bevacizumab ( $7.5 \mathrm{mg} / \mathrm{kg}$ bw i.v.). These patients received six cycles of $\mathrm{CP}$ chemotherapy and received concomitant bevacizumab from the second to the 6th cycle. After the 6th CP cycle, bevacizumab was continued as a standalone treatment until the maximum of 18 cycles or until disease recurrence. Average BMI was similar between the two groups (baseline demographic information included in Table 1). All patients were prescribed low molecular weight (LMW) heparin during the first 6 weeks after surgery. The dosage of LMWH was prescribed based on the patient weight.

Patients from both groups underwent suboptimal debulking, and the residual disease was very similar in all cases $(1-5 \mathrm{~cm}$ found in abdomen and pelvic organs, such as large intestine, urinary bladder, liver). Control group patients were initially qualified for bevacizumab treatment, but were later excluded as a result of consent withdrawal (one patient) or various medical reasons, including Zubrod functional score higher than 1 (two patients), ulcerative colitis (one patient), slow post-surgical wound healing (five patients), age above 65 years (nine patients), elevated coagulological parameters (namely APTT, one patient), PT and INR (seven patients),
Table 1 Baseline demographic information for the study and control group

\begin{tabular}{lll}
\hline Gender & $\begin{array}{l}\text { CP + bevacizumab group } \\
\text { Female }-31(100 \%)\end{array}$ & $\begin{array}{l}\text { CP group } \\
\text { Female }-26(100 \%)\end{array}$ \\
\hline Race or ethnicity & Caucasian $-31(100 \%)$ & Caucasian $-26(100 \%)$ \\
Mean age in years & 58.77 & 60.17 \\
BMI before chemotherapy & 24.1 & 24.7 \\
\hline
\end{tabular}


but not D-dimer concentration, elevated bilirubin (twice the normal value, one patient), elevated transaminases (five times the normal value, one patient), elevated creatinine (one patient), venous thrombosis in patient's history or oral anticoagulant use (one patient). Some of the patients met several of the exclusion criteria. The first cycle of chemotherapy was administered approximately 21 days after the surgery and consisted of CP only in both groups. Patients were administered chemotherapy every 21 days.

\subsection{Methods}

Patients were divided according to Khorana score into VTE risk groups based on parameters assessed approximately 21 days after the surgery and right before the first round of chemotherapy. The influence of bevacizumab treatment on VTE was investigated against the initial pretreatment VTE risk. VTE risk and laboratory tests (total leukocytes, platelet count, hemoglobin, APTT, prothrombin time, INR, fibrinogen levels, D-dimer concentration) were measured in both groups after each round of chemotherapy. The aim was to assess the influence of bevacizumab treatment on these factors. Changes in abovementioned parameters prior to thromboembolic events were also evaluated.

VTE was diagnosed by a vascular surgeon based on clinical signs, such as extremity pain, swelling, and bruising, with the addition of Doppler ultrasound imaging. No routine screening was performed. In case of VTE suspicion, a vascular surgeon consultation was scheduled for the diagnosis to be confirmed.

All parameters were assessed by qualified specialists in laboratory medicine and measured in the Central Laboratory of the hospital where the study was performed. Total leukocytes were assessed with fluorescence flow cytometry in optic detector. Platelet count was measured with impedance/ conductometric method. Hemoglobin was assessed with HGB detector using sodium lauryl sulfate (SLS) with a photometric method. Activated partial thromboplastin time (APTT) measurement was based on incubation of plasma with standardized phospholipids amount and negatively charged contact activator, which lead to internal coagulation pathway activation. After incubation at $37^{\circ} \mathrm{C}$ for a standardized time, calcium was added and the time required for clot formation was measured. To assess prothrombin time (PT), a reagent with calcium thromboplastin was added to citrate plasma samples, which activates a cascade leading to fibrin clot formation. Time between reagent addition and clot formation was measured and compared to the time the same process took for the reference plasma. INR (International Normalized Ratio) was calculated based on these results. Fibrinogen in diluted sample is converted into fibrin through adding excess of thrombin. Clotting time was measured. Logarithm of clotting time is in inverse proportion to logarithm of fibrinogen concentration. A reference curve was established based on reference plasma clotting time with various dilution ratios measurements. Fibrinogen concentration in patients samples was calculated from clotting time according to the reference curve. D-dimer concentration was assessed with the use of a special reagent, a suspension of polystyrene latex normalized particles covered with a monoclonal antibody against D-dimer domains which are found in soluble fibrin degradation products. After agglutination, D-dimer concentration was assessed by measuring the degree of light transmission in $405 \mathrm{~nm}$ (turbidimetric method).

\subsection{Statistical Evaluation of Data}

Data were analyzed using Statistica 12 software (Statistica, Tulsa, OK, USA). Statistical significance alpha was set to $5 \%(p=0.05)$. For categorical variables, a $\chi^{2}$ test was used to analyze the dependence of bevacizumab treatment to VTE occurrence. In this test, VTE events were included as dependent data and bevacizumab treatment as independent data. Continuous variables with non-normally distributed data were compared using Mann-Whitney test to distinguish VTE risk factors. VTEs were again included as dependent data. Total leukocytes, platelet count, hemoglobin, APTT, prothrombin time, INR, fibrinogen levels, D-dimer concentration were included as independent data. Continuous variables with nonnormally distributed data with three or more variables were compared using Friedman test to check the changes of all studied parameters during chemotherapy administrations and to check how all the parameters changed in patients with and without VTE. To evaluate how all the investigated parameters changed before and during venous thrombosis events, continuous variables with non-normally distributed data with two variables were analyzed with a Wilcoxon test. Total leukocytes, platelet count, hemoglobin, APTT, prothrombin time, INR, fibrinogen levels, D-dimer concentration were dependent data in Friedman test and Wilcoxon test. For cut-off point detection a ROC curve was used to establish the best D-dimer value in detecting VTE in our study. A ROC curve was made in PQStat software, version 1.6.2 (PQStat Software, Poznan, Poland).

\section{Results}

Among the 57 patients included in the study, five (8.77\%) had a thromboembolic event (VTE patients). The control group (CP) contained 26 individuals. The study group (CP + bevacizumab) comprised 31 patients. Only patients in the group receiving bevacizumab experienced VTE $(p=0.03)$. All parameters were analyzed starting from approximately day 21 post surgery, before the first chemotherapy administration, and before each next therapy cycle. The mean time of 
VTE occurrence was the 13th cycle of chemotherapy, during bevacizumab administration only.

Median BMI before chemotherapy for patients affected with VTE was 27.2 compared to 23.3 for patients without a thromboembolic event ( $p=0.005$ ). Median D-dimer concentration before treatment was 3132.5 for VTE patients vs. 956.43 for non-VTE patients $(p=0.0007)$. No other parameters (hemoglobin concentration, total leukocytes, platelet count) were found to be of significance as risk factors for the prediction of thromboembolic events (Table 2).

Table 3 shows how the patients were divided into groups according to the Khorana VTE risk score. The use of bevacizumab was associated with a statistically non-significant increase in VTE risk in the high-risk group compared to the medium risk group $(\mathrm{RR}=1.82$; 95\% confidence interval 0.35 9.42). No patients with low risk score experienced VTE.

Changes in laboratory findings and assessment of other VTE risk factors in the course of first four therapy cycles for both grous are shown in Tables 4 and 5 .

Mean number of bevacizumab doses was higher in patients with vs. without VTE (12.0 vs 7.6). For each patient, values of all parameters registered before chemotherapy administrations were taken into consideration. All patients in the CP + bevacizumab group had at least four chemotherapy administrations (some patients had only four administrations of bevacizumab and for others it was a continuation of a previous chemotherapy regimen). Patients in the CP group had at least three chemotherapy administrations. Therefore in this analysis, parameters measured before chemotherapy and during 4 chemotherapy administrations in patients from CP + bevacizumab scheme and 3 chemotherapy administrations in patients with $\mathrm{CP}$ only were taken into consideration in order to have the same number of measurements in each patient. Friedman test was used to determine that during the first four administrations of $\mathrm{CP}+$ bevacizumab chemotherapy the following changes occured: reduction in D-dimer concentration, platelet count and prothrombin time and increase in leucocytes
(Table 4). During the first three administrations of the CP only regimen, D-dimer concentration, hemoglobin and platelet count decreased and differed from one chemotherapy cycle to another (Table 5).

In a similar way, we analyzed alterations in laboratory findings in the first chemotherapy cycles within a group of patients affected with VTE (Table 6) compared to those that had no thromboembolic incident (Table 7). Friedman test was used to determine that during the first four chemotherapy administrations in patients with VTE there was a reduction in D-dimer concentration and extension of APTT (Table 6), while in individuals not affected with VTE increase in leukocyte and platelet count was observed (Table 7).

Figure 1 shows how the investigated parameters changed right before and during VTE. The D-dimer concentration (ng/ $\mathrm{mL}$ ) measured at a chemotherapy cycle immediately before the VTE increased on average from 1665 to 9000 during VTE $(p=0.03)$. Similarly, APTT measured immediately before the VTE dropped from $28.85 \mathrm{~s}$ to $20.9 \mathrm{~s}$ during VTE, (median 28.7 vs. $21.5, p=0.04$, Wilcoxon test). There were no significant differences in other parameters measured in this study.

A cutoff point for D-dimer value was set at $485 \mathrm{ng} / \mathrm{mL}$ based on a ROC curve (Fig. 2). It has a $94 \%$ sensitivity and $36 \%$ specificity for detecting VTE events in ovarian cancer patients who underwent chemotherapy.

\section{Discussion}

There are reports stating that some types of chemotherapy increase the VTE risk more than other regimens [16]. Our study suggests that bevacizumab increases the risk of thrombosis, which is consistent with other existing reports [11]. However, many studies (and large meta-analyses) have shown that bevacizumab does not increase risk of venous thromboembolism, only the risk of arterial thromboembolism [9, 12], while in our study all analyzed events concerned VTE.
Table 2 Median values of variables analyzed before the start of chemotherapy in patients with and without VTE

\begin{tabular}{lccl}
\hline Parameters before chemotherapy & Patients with VTE & Patients without VTE & $\mathrm{p}$ \\
\hline BMI & 27.2 & 23.3 & 0.005 \\
D-dimers, ng/mL & 3132.5 & 956.43 & 0.007 \\
Leukocytes, G/L & 7.88 & 6.58 & 0.9 \\
PLT, G/L & 321.0 & 288.92 & 0.87 \\
Hb, mmol/L & 6.95 & 6.99 & 0.1 \\
APTT, s & 25.5 & 29.71 & 0.37 \\
PT, s & 13.03 & 12.14 & 0.37 \\
INR & 1.02 & 1.02 & 1.0 \\
Fibrinogen, g/L & 4.2 & 3.56 & 1.0 \\
\hline
\end{tabular}

PLT blood platelets, $\mathrm{Hb}$ hemoglobin, APTT activated partial thromboplastin time, PT prothrombin time, INR international normalized ratio 
Table 3 Patient stratification according to Khorana VTE risk score

\begin{tabular}{lllllll}
\hline Risk group & \multicolumn{2}{l}{ High risk } & & \multicolumn{2}{l}{ Medium risk } \\
\cline { 2 - 3 } \cline { 5 - 6 } Therapy & $\mathrm{CP}+$ bevacizumab & $\mathrm{CP}$ & & $\mathrm{CP}+$ bevacizumab & $\mathrm{CP}$ \\
\hline Number of patients & 14 & 14 & 17 & 12 \\
Patients with VTE & 3 & 0 & 2 & 0 \\
\hline
\end{tabular}

VTE venous thromboembolism, CP paclitaxel and carboplatin

Sixteen percent of our patients treated with $\mathrm{CP}+$ bevacizumab chemotherapy regimen had a venous thrombosis event, whereas none of the patients treated with standard, first line CP chemotherapy suffered a similar event. There were no patients with arterial thromboembolism.

In the AURELIA study [17] there were less VTEs in patients treated with bevacizumab (3\%) compared to those treated with chemotherapy only (4\%). Two percent of women treated with bevacizumab suffered from arterial thrombosis, but none of the patients without bevacizumab treatment did.

The cause of VTE risk increase in our study is uncertain. However, patients from our study had more risk factors for venous thrombosis compared to arterial thrombosis, in particular those mentioned in Virchow's triad as hypercoagulability. It is possible that hypercoagulability in our patients could be the result of residual disease causing the release of tissue factor (TF) by tumor cells. It could also be caused by bevacizumab administration, which could impair the regeneration of endothelium, cause exposition of subendothelial collagen, and the activation of the extrinsic coagulation pathway as a result of the tissue factor release from the vascular wall [18]. In contrast to venous thrombosis, activation of coagulation factors does not appear to play a major role in arterial thrombosis, as these factors are likely to be removed by the high flow in the arterial system.
Vessel wall damage is a major risk factor for arterial thrombosis, by inducing turbulence and altered blood flow, which allows platelet adhesion. In our patients this mechanism of thrombosis induction is less probable. Only few patients in our study suffered from hypertension and diabetes and they were not the same patients who suffered from a VTE.

It was confirmed that elevated D-dimer [19] and BMI [20] values are indeed major risk factors of thromboembolic disease. BMI, as a parameter that is modifiable, deserves particular attention. Proper diet and achieving a healthy body mass would, with high probability, decrease the risk of venous thrombosis events. According to the Khorana risk score [6], a BMI of $35 \mathrm{~kg} / \mathrm{m}^{2}$ is a VTE risk factor. Our study shows a much lower BMI value for VTE occurrence, which remains in accordance with previously published research [21, 22].

All patients with VTE had elevated D-dimer levels prior to the first cycle of chemotherapy. It should be noted that this is not an exclusion criteria for bevacizumab treatment. D-dimer levels of these patients increased during longer treatment and did so quite significantly, immediately prior and during the VTE event. D-dimers, therefore, possess both diagnostic and prognostic value for thromboembolic events, which was also demonstrated in another study [23].

CP chemotherapy itself was shown not to increase the risk of VTE in our study, especially considering the fact that both groups received no more than six $\mathrm{CP}$ cycles. No publications were found to state such a hypothesis, either. Consistently, one study suggested that low doses of paclitaxel and carboplatin were safe for patients with coexisting ovarian cancer and thromboembolic disease [24].

A limitation of our study is that patients were qualified to the CP group because they did not meet certain criteria for bevacizumab therapy, which might have introduced some bias. Ideally, a randomized trial of $\mathrm{CP}$ vs. $\mathrm{CP}+$ bevacizumab

Table 4 Mean values and standard deviations of variables analyzed before and during chemotherapy in CP + bevacizumab patients

\begin{tabular}{|c|c|c|c|c|c|c|}
\hline \multirow[t]{2}{*}{ Parameter } & \multicolumn{6}{|l|}{$\mathrm{CP}+$ bevaciumab } \\
\hline & Before chemotherapy & I administration & II administration & III administration & IV administration & $\mathrm{p}$ \\
\hline D-dimers, $\mathrm{ng} / \mathrm{mL}$ & $1747.72 \pm 1772$ & $1144.27 \pm 1193.17$ & $1126.27 \pm 1281.18$ & $990 \pm 1155.91$ & $942 \pm 1127.77$ & 0.02 \\
\hline Leukocytes, G/L & $6.9 \pm 2.39$ & $7.51 \pm 2.24$ & $8.28 \pm 2.33$ & $6.79 \pm 4.02$ & $6.87 \pm 2.3$ & 0.04 \\
\hline PLT, G/L & $296.47 \pm 104.33$ & $309.24 \pm 120.41$ & $255.24 \pm 64.32$ & $193.35 \pm 68.04$ & $279.88 \pm 130.8$ & 0.001 \\
\hline $\mathrm{Hb}, \mathrm{mmol} / \mathrm{L}$ & $6.98 \pm 0.67$ & $7.02 \pm 0.7$ & $6.94 \pm 0.51$ & $6.89 \pm 0.66$ & $6.82 \pm 0.54$ & 0.15 \\
\hline APTT, s & $28.66 \pm 2.42$ & $30.21 \pm 1.84$ & $29.82 \pm 3.04$ & $29.63 \pm 2.94$ & $29.54 \pm 2.5$ & 0.56 \\
\hline PT, s & $12.62 \pm 1.37$ & $12.2 \pm 1.18$ & $11.94 \pm 1.1$ & $11.77 \pm 1.15$ & $11.8 \pm 1.25$ & 0.03 \\
\hline INR & $1.07 \pm 0.09$ & $1.92 \pm 3.02$ & $1.03 \pm 0.06$ & $1.04 \pm 0.09$ & $1.03 \pm 0.07$ & 0.13 \\
\hline Fibrinogen, $\mathrm{g} / \mathrm{L}$ & $3.7 \pm 1.04$ & $3.42 \pm 0.83$ & $3.4 \pm 0.99$ & $3.41 \pm 0.99$ & $3.56 \pm 1.25$ & 0.19 \\
\hline
\end{tabular}

PLT blood platelets, $\mathrm{Hb}$ hemoglobin, APTT activated partial thromboplastin time, PT prothrombin time, INR international normalized ratio 
Table 5 Mean values and standard deviations of variables analyzed before and during chemotherapy in CP patients

\begin{tabular}{|c|c|c|c|c|c|}
\hline \multirow[t]{2}{*}{ Parameter } & \multicolumn{5}{|l|}{$\mathrm{CP}$} \\
\hline & Before chemotherapy & I administration & II administration & III administration & $\mathrm{p}$ \\
\hline D-dimers, $\mathrm{ng} / \mathrm{mL}$ & $1655.75 \pm 1550$ & $1287.33 \pm 1354.18$ & $1236.35 \pm 1322.53$ & $955 \pm 1098.8$ & 0.03 \\
\hline Leukocytes, G/L & $8.29 \pm 3.08$ & $7.88 \pm 2.04$ & $7.47 \pm 4.9$ & $7.08 \pm 2.01$ & 0.17 \\
\hline PLT, G/L & $427.2 \pm 195.38$ & $305.56 \pm 174.83$ & $300.36 \pm 171.86$ & $211.12 \pm 77.9$ & 0.0001 \\
\hline $\mathrm{Hb}, \mathrm{mmol} / \mathrm{L}$ & $7.37 \pm 0.84$ & $6.95 \pm 0.69$ & $6.8 \pm 0.69$ & $6.47 \pm 0.6$ & 0.0001 \\
\hline APTT, s & $29.52 \pm 2.15$ & $28.75 \pm 2.54$ & $30.3 \pm 2.04$ & $29.85 \pm 3.15$ & 0.43 \\
\hline PT, s & $12.44 \pm 1.78$ & $12.53 \pm 1.35$ & $12.57 \pm 1.2$ & $12.7 \pm 1.9$ & 0.34 \\
\hline INR & $1.03 \pm 0.02$ & $1.02 \pm 0.05$ & $1.9 \pm 0.08$ & $1.02 \pm 0.06$ & 0.63 \\
\hline Fibrinogen, $\mathrm{g} / \mathrm{L}$ & $3.9 \pm 1.06$ & $3.62 \pm 0.9$ & $3.55 \pm 1.02$ & $3.5 \pm 0.86$ & 0.3 \\
\hline
\end{tabular}

PLT blood platelets, $\mathrm{Hb}$ hemoglobin, APTT activated partial thromboplastin time, PT prothrombin time, INR international normalized ratio

stratified with studied variables would potentially address this point. In the light of current knowledge supporting beneficial influence of $\mathrm{CP}+$ bevacizumab therapy such a study model might raise ethical concerns. Even though we are not able to draw final conclusions about unbiased comparison of CP vs. $\mathrm{CP}+$ bevacizumab therapy, our study demonstrates that the current form of $\mathrm{CP}+$ bevacizumab therapy bears a substantial risk of VTE.

It should be noted that neither $\mathrm{CP}$ alone nor $\mathrm{CP}+$ bevacizumab increased the VTE risk via Khorana risk score parameters elevation (leukocytes, platelet count, $\mathrm{Hb}$ ) in our study. Chemotherapy causes all parameters to decrease due to bone marrow suppression. Therefore, these parameters might be useful only in assessing the risk of thrombosis in patients before chemotherapy, not during treatment. The decrease in their levels during chemotherapy does not indicate the real decrease of thromboembolism risk. The risk can be increased by other factors, such as other mechanisms of chemotherapy action.

It should be noted that during the first four bevacizumab administrations, there was a significant increase in leukocyte count due to unknown reasons, and this was the only change in parameters which could increase the VTE risk according to the Khorana score. This change, however, was not observed in patients with VTE. In both the bevacizumab + CP and the CP group, there was a significant decrease in D-dimer concentration, which may be explained by previous surgical removal of cancer, which normally produces cytokines and other procoagulative substances.

In patients with VTE a significant decrease in D-dimer concentration and an increase of APTT at the beginning of chemotherapy cycle was observed. APTT, lower before surgery and chemotherapy, increased after the surgery and chemotherapy administration. These changes could be also explained by a decrease in procoagulative effects after cancer surgery. However, these results should be considered with caution, because the analysis was made on only five patients with VTE.

It was observed that bevacizumab could probably influence the coagulological parameters used to assess the extrinsic coagulation pathway. The average for

Table 6 Mean values and standard deviations of variables analyzed before and during chemotherapy in patients with VTE

\begin{tabular}{|c|c|c|c|c|c|c|}
\hline \multirow[t]{2}{*}{ Parameter } & \multicolumn{6}{|l|}{ Patients with VTE } \\
\hline & Before chemotherapy & I administration & II administration & III administration & IV administration & $\mathrm{p}$ \\
\hline D-dimers, $\mathrm{ng} / \mathrm{mL}$ & $3132.5 \pm 2390$ & $1963 \pm 1734.1$ & $1965.3 \pm 1904.5$ & $1705.2 \pm 1739.9$ & $1475.7 \pm 1707.5$ & 0.04 \\
\hline Leukocytes, G/L & $7.88 \pm 1.92$ & $8.8 \pm 1.86$ & $7.99 \pm 2.51$ & $5.87 \pm 1.26$ & $6.97 \pm 2.98$ & 0.46 \\
\hline PLT, G/L & $321.0 \pm 95.34$ & $264.25 \pm 77.25$ & $271.75 \pm 34.4$ & $157.25 \pm 75.23$ & $244.00 \pm 85.26$ & 0.14 \\
\hline $\mathrm{Hb}, \mathrm{mmol} / \mathrm{L}$ & $6.95 \pm 0.74$ & $7.03 \pm 0.59$ & $6.8 \pm 0.49$ & $6.65 \pm 0.75$ & $7.1 \pm 0.47$ & 0.55 \\
\hline APTT, s & $25.5 \pm 0.8$ & $29.5 \pm 3.3$ & $28.4 \pm 1.8$ & $26.7 \pm 3.1$ & $28 \pm 2.3$ & 0.04 \\
\hline PT, s & $13.03 \pm 1.63$ & $12.23 \pm 1.4$ & $12.07 \pm 1.33$ & $11.9 \pm 1.13$ & $12.07 \pm 2.02$ & 0.75 \\
\hline INR & $1.02 \pm 0.02$ & $1.03 \pm 0.06$ & $1.02 \pm 0.02$ & $1.02 \pm 0.02$ & $1.00 \pm 0.03$ & 0.92 \\
\hline Fibrinogen, $g / L$ & $4.2 \pm 0.65$ & $3.7 \pm 1.1$ & $3.5 \pm 0,51$ & $3.6 \pm 0.27$ & $3.6 \pm 1.37$ & 0.44 \\
\hline
\end{tabular}

PLT blood platelets, $\mathrm{Hb}$ hemoglobin, APTT activated partial thromboplastin time, PT prothrombin time, INR international normalized ratio 
Table 7 Mean values and standard deviations of variables analyzed before and during chemotherapy in patients without VTE

\begin{tabular}{|c|c|c|c|c|c|c|}
\hline \multirow[t]{2}{*}{ Parameter } & \multicolumn{6}{|l|}{ Patients without VTE } \\
\hline & Before chemotherapy & I administration & II administration & III administration & IV administration & $\mathrm{p}$ \\
\hline D-dimers, $\mathrm{ng} / \mathrm{mL}$ & $956.43 \pm 606.88$ & $676.43 \pm 408.6$ & $646.86 \pm 430.34$ & $581.29 \pm 420.95$ & $637.00 \pm 602.92$ & 0.22 \\
\hline Leukocytes, G/L & $6.58 \pm 2.52$ & $7.08 \pm 2.26$ & $8.37 \pm 2.38$ & $7.09 \pm 4.6$ & $6.84 \pm 2.2$ & 0.04 \\
\hline PLT, G/L & $288.92 \pm 109.45$ & $323.07 \pm 130.22$ & $250.15 \pm 71.43$ & $204.46 \pm 64.73$ & $290.92 \pm 142.94$ & 0.006 \\
\hline $\mathrm{Hb}, \mathrm{mmol} / \mathrm{L}$ & $6.99 \pm 0.67$ & $7.02 \pm 0.76$ & $6.98 \pm 0.53$ & $6.96 \pm 0.64$ & $6.73 \pm 0.54$ & 0.16 \\
\hline APTT, s & $29.71 \pm 1.7$ & $30.43 \pm 1.31$ & $30.28 \pm 3.29$ & $30.59 \pm 2.29$ & $30.03 \pm 2.49$ & 0.85 \\
\hline PT, s & $12.14 \pm 1.71$ & $12.19 \pm 1.19$ & $12.17 \pm 1.16$ & $11.83 \pm 1.18$ & $11.99 \pm 1.35$ & 0.57 \\
\hline INR & $1.02 \pm 0.07$ & $2.19 \pm 3.49$ & $1.03 \pm 0.02$ & $1.03 \pm 0.05$ & $1.03 \pm 0.04$ & 0.75 \\
\hline Fibrinogen, $g / L$ & $3.56 \pm 1.14$ & $3.34 \pm 0.78$ & $3.36 \pm 1.12$ & $3.35 \pm 1.15$ & $3.54 \pm 1.3$ & 0.45 \\
\hline
\end{tabular}

PLT blood platelets, $\mathrm{Hb}$ hemoglobin, APTT activated partial thromboplastin time, PT prothrombin time, INR international normalized ratio

prothrombin time during bevacizumab treatment was reduced to $11.93 \pm 1.37 \mathrm{~s}$, below the reference value of 12-16 s [25]. The prothrombotic effect of bevacizumab is caused by its suppressive activity of VEGF. The drug impairs the regeneration of endothelium, causes exposition of subendothelial collagen, and activation of the extrinsic coagulation pathway as a result of the tissue factor release from the vascular wall [18].

The PROTECHT study (Prophylaxis of Thromboembolism During Chemotherapy) emphasized the importance of VTE prevention for chemotherapy patients [26]. Our research tried to determine the best moment for prophylaxis implementation, i.e. at what point of treatment the highest VTE rate occurs. We observed that INR and prothrombin time (PT) decreased consecutively prior to a thromboembolic event, indicating the activation of the extrinsic coagulation pathway related to the mode of action of bevacizumab. We noted also a tendency towards an increase of leukocyte count in each patient before the VTE. We found no evidence for an activation of the intrinsic coagulation pathway by bevacizumab. The APTT remained unaltered before VTE, while its reduction, observed during thromboembolic events, might be expected regardless of the cause for VTE.

In our study, the mean number of bevacizumab doses was higher in patients with vs. without VTE (12.0 vs. 7.6) suggesting possible cumulative doses effect. This is in accordance with a different study, which also revealed that the mean number of bevacizumab doses was higher in the group with thromboembolism (12.6 vs. 9.0) [27]. It is possible that thromboembolic prophylaxis could be helpful during long-term bevacizumab administration. However, while considering thromboembolic prophylaxis, bleeding risk should be assessed as well and balance between hemorrhage and thromboembolism should be maintained on safety level. Further research is necessary.
The cutoff value for D-dimers established in our study $(485 \mathrm{ng} / \mathrm{mL})$ has a very high $(94 \%)$ sensitivity for VTE prediction and detection in ovarian cancer patients treated with chemotherapy and confirms the high diagnostic value of the existing laboratory norm of $500 \mathrm{ng} / \mathrm{mL}$.

It should be noted that the group in our study was too small to consider it a sufficient representation of the ovarian cancer patient population: six times smaller than the AURELIA study group. This could impact the outcomes, e.g. dividing patients according to Khorana score probably would have been statistically significant if there were more patients and more thrombosis events. To confirm our findings, further research on a larger scale is necessary. Furthermore, control group patients were excluded from bevacizumab treatment for various medical reasons, so they could be more or less predisposed to thrombosis compared to the study group. There were major differences in patient characteristics between the groups especially with age and laboratory tests. We cannot state that bevacizumab administration was the only factor influencing thrombosis events in the research, because patient groups were not homogenous as far as other thrombosis risk factors are concerned.

Our study suggests that patients treated with bevacizumab are more susceptible to thromboembolic events than women undergoing standard first line chemotherapy and as such should be more carefully observed during therapy, including more attentive observation of VTE indicators and coagulological parameters.

An increased rate of thromboembolic events in patients with obesity suggests more attention should be directed into maintaining correct body weight through healthy lifestyle promotion. Balanced diet and dietary consultation before and during chemotherapy could play a role in venous thrombosis prevention, as should moderate amount of exercise many cancer patients will be 


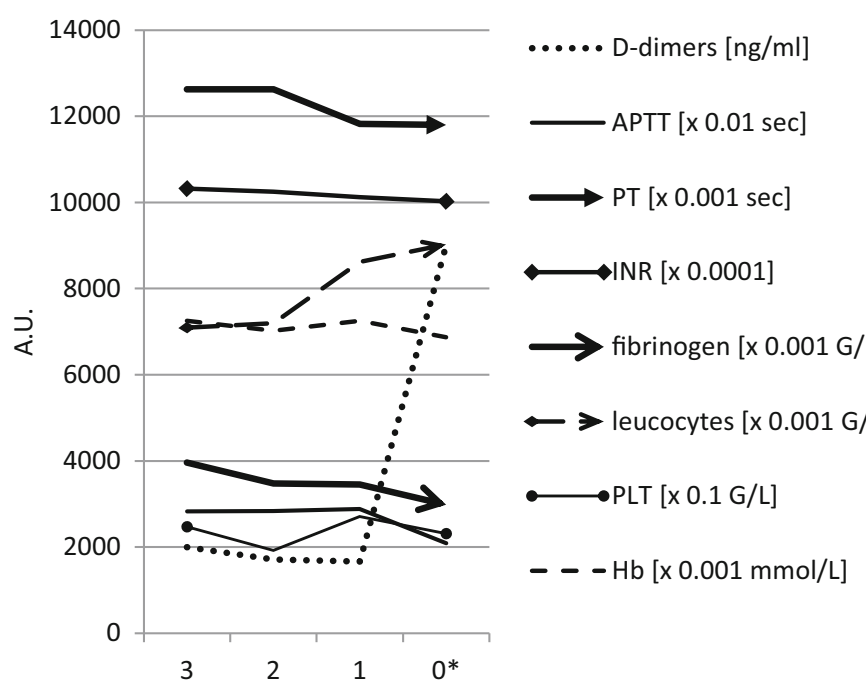

consecutive chemotherapy administrations before thromboembolic incident

Fig. 1 Comparison of the mean values of all variables analyzed three, two, and one chemotherapy cycle before the thromboembolic event and during the event. *0 (zero) indicates event. PLT blood platelets, Hb hemoglobin, APTT activated partial thromboplastin time, PT prothrombin time, INR international normalized ratio

able to perform. It would also positively influence patients' quality of life.

Bevacizumab probably has an influence on the extrinsic coagulation pathway attributed to tissue factor $(\mathrm{TF})$ release from the vascular wall [28]. Physiologically, TF is suppressed by the Tissue Factor Pathway Inhibitor (TFPI) produced by the endothelium [29]. Bevacizumab restricts the proliferation of endothelium and therefore probably triggers TFPI deficiency.

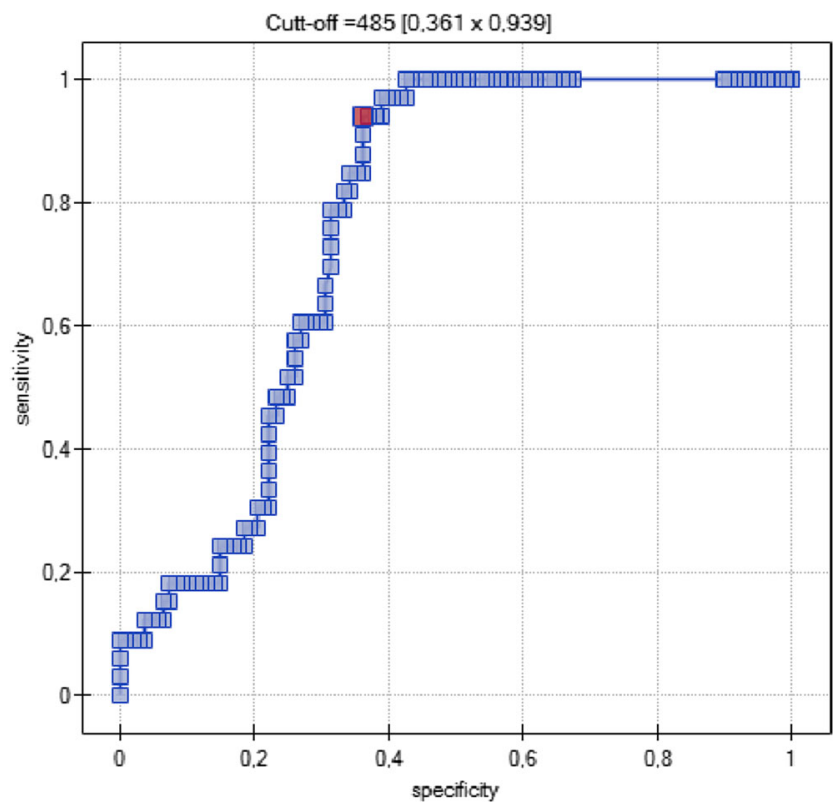

Fig. 2 ROC curve for D-dimer for detecting VTE events

\section{Conclusions}

Ovarian cancer is a highly heterogeneous disease, which raises a need for personalized therapies. PARP inhibitors treatment in patients with BRCA mutations is a good example of adjusting management in a subgroup of patients. Our work demonstrates that prediction of a serious side effect of bevacizumab therapy, VTE is dependent on BMI value and D-Dimer level as measured prior to the onset of chemotherapy. This knowledge, along with other studies, may help to personalize management in ovarian cancer patients treated with antiangiogenic therapy. Bevacizumab administration should be considered individually in every patient, considering risk factors for VTE, including obesity and elevated Ddimer concentration.

Acknowledgements Piotr Majek from Faculty of Physics, Adam Mickiewicz University, Poznań, Poland made the tables and figures of this manuscript.

\section{Compliance with Ethical Standards}

Funding This study received no funding.

Conflict of Interest Anna Kuk, Magdalena Magnowska, M.D., Ph.D, Wiktor Suchy, M.D., Joanna Swierczynska, Mikolaj Piotr Zaborowski, M.D., Prof. Michal Gaca, and Prof. Ewa Nowak-Markwitz declare that they have no conflict of interest.

Ethical Approval For this type of study formal consent is not required.

Open Access This article is distributed under the terms of the Creative Commons Attribution-NonCommercial 4.0 International License (http:// creativecommons.org/licenses/by-nc/4.0/), which permits any noncommercial use, distribution, and reproduction in any medium, provided you give appropriate credit to the original author(s) and the source, provide a link to the Creative Commons license, and indicate if changes were made.

\section{References}

1. Rodrigues CA, Ferrarotto R, Kalil FR, Novis YA, Hoff PM. Venous thromboembolism and cancer: a systematic review. J Thromb Thrombolysis. 2010;30(1):67-78.

2. Lyman GH. Venous thromboembolism in the patient with cancer: focus on burden of disease and benefits of thromboprophylaxis. Cancer. 2011;117(7):1334-49.

3. Fennerty A. Venous thromboembolic disease and cancer. Postgrad Med J. 2006;82(972):642-8.

4. Lee AY, Levine MN, Butler G, Webb C, Costantini L, Gu C, Julian JA. Incidence, risk factors, and outcomes of catheter-related thrombosis in adult patients with cancer. J Clin Oncol. 2006;24(9):1404-8.

5. Blom JW, Vanderschoot JP, Oostindiër MJ, Osanto S, van der Meer FJ, Rosendaal FR. Incidence of venous thrombosis in a large cohort of 66,329 cancer patients: results of a record linkage study. J Thromb Haemost. 2006;4(3):529-35. 
6. Khorana AA, Kuderer NM, Culakova E, Lyman GH, Francis CW. Development and validation of a predictive model for chemotherapyassociated thrombosis. Blood. 2008;111(10):4902-7.

7. Burger RA, Brady MF, Bookman MA, et al. Incorporation of bevacizumab in the primary treatment of ovarian cancer. N Engl J Med. 2011;365(26):2473-83.

8. Burger RA, Sill MW, Monk BJ, Greer BE, Sorosky JI. Phase II trial of bevacizumab in persistent or recurrent epithelial ovarian cancer or primary peritoneal cancer: a Gynecologic oncology group study. J Clin Oncol. 2007;25:5165-71.

9. Cannistra SA, Matulonis UA, Penson RT, et al. Phase II study of bevacizumab in patients with platinum-resistant ovarian cancer or peritoneal serous cancer. J Clin Oncol 2007;25:5180-5186[erratum]. J Clin Oncol. 2008;26:1773.

10. Perren TJ, Swart AM, Pfisterer J, et al. A phase 3 trial of bevacizumab in ovarian cancer. N Engl J Med. 2011;365(26): 2484-96.

11. Nalluri SR, Chu D, Keresztes R, Zhu X, Wu S. Risk of venous thromboembolism with the angiogenesis inhibitor bevacizumab in cancer patients: a meta-analysis. JAMA. 2008;300(19):2277-85.

12. Scappaticci FA, Skillings JR, Holden SN, et al. Arterial thromboembolic events in patients with metastatic carcinoma treated with chemotherapy and bevacizumab. JNCI J Natl Cancer Inst. 2007;99(16):1232-9.

13. Hurwitz HI, Saltz LB, Van Cutsem E, et al. Venous thromboembolic events with chemotherapy plus bevacizumab: a pooled analysis of patients in randomized phase II and III studies. J Clin Oncol. 2011;29(13):1757-64.

14. Yu J, Cao XF, Zheng Y, et al. Anti-VEGF therapy with bevacizumab-limited cardiovascular toxicity. Asian Pac J Cancer Prev. 2014;15(24):10769-72.

15. Patel JN, Jiang C, Hertz DL, et al. Bevacizumab and the risk of arterial and venous thromboembolism in patients with metastatic, castration-resistant prostate cancer treated on cancer and leukemia group B (CALGB) 90401 (alliance). Cancer. 2015;121(7):102531.

16. Barni S, Labianca R, Agnelli G, et al. Chemotherapy-associated thromboembolic risk in cancer outpatients and effect of nadroparin thromboprophylaxis: results of a retrospective analysis of the PROTECHT study. J Transl Med. 2011;9:179.

17. Pujade-Lauraine E, Hilpert F, Weber B, et al. Bevacizumab combined with chemotherapy for platinum-resistant recurrent ovarian cancer: the AURELIA open-label randomized phase III trial. J Clin Oncol. 2014;32(13):1302-8.

18. Kilickap S, Abali H, Celik I. Bevacizumab, bleeding, thrombosis, and warfarin. J Clin Oncol. 2003;21(18):3542-6.

19. Pulivarthi S, Gurram MK. Effectiveness of d-dimer as a screening test for venous thromboembolism: an update. N Am J Med Sci. 2014;6(10):491-9.

20. Mereu L, Tateo S, Klersy C, Martinotti Gabellotti E, Polatti F. Stratification of venous thromboembolism risk in ovarian cancer patients during chemotherapy. Int J Gynecol Cancer. 2009;19(1): 79-83.

21. Pant A, Liu D, Schink J, Lurain J. Venous thromboembolism in advanced ovarian cancer patients undergoing frontline adjuvant chemotherapy. Int J Gynecol Cancer. 2014;24(6):997-1002.

22. Abu Saadeh F, Norris L, O'Toole S, Gleeson N. Venous thromboembolism in ovarian cancer: incidence, risk factors and impact on survival. Eur J Obstet Gynecol Reprod Biol. 2013;170(1):214-8.

23. Folsom AR, Alonso A, George KM, RoeCPer NS, Tang W, Cushman M. Prospective study of plasma D-dimer and incident venous thromboembolism: the atherosclerosis risk in communities (ARIC) study. Thromb Res. 2015;136(4):781-5.

24. Tabata T, Tanida K, Umekawa T, et al. Weekly low-dose paclitaxel and carboplatin therapy in gynecological cancer patients with venous thrombosis. Anticancer Res. 2008;28(6B):3971-5.

25. Andrzej Szczeklik. Choroby wewnętrzne. Przyczyny, rozpoznanie i leczenie, tom I. Kraków, Wydawnictwo Medycyna Praktyczna; 2005:30-31.

26. Agnelli G, Gussoni G, Bianchini C, Verso M, Tonato M. A randomized double-blind placebo-controlled study on Nadroparin for prophylaxis of thromboembolic events in cancer patients receiving chemotherapy: the PROTECHT study. Blood. 2008;112(11):6.

27. I. S. Yu, Winson Y. Cheung. Risk of arterial (ATE) and venous thromboembolism (VTE) in a population-based cohort of bevacizumab-treated metastatic colorectal cancer (mCRC) patients. J Clin Oncol 31;2013(suppl 4; abstr 545).

28. Mackman N. The role of tissue factor and factor VIIa in hemostasis. Anesth Analg. 2009;108(5):1447-52.

29. Bajaj MS, Kuppuswamy MN, Saito H, Spitzer SG, Bajaj SP. Cultured normal human hepatocytes do not synthesize lipoprotein- associated coagulation inhibitor: evidence that endothelium is the principal site of its synthesis. Proc Natl Acad Sci U S A. 1990;87(22):8869-73. 